\title{
Adaptación y propiedades psicométricas del inventario de depresión de beck (bdi-ii) en adolescentes ecuatorianos
}

\section{Adaptation and psychometric properties of the beck depression inventory (bdi-ii) in ecuadorian adolescents}

\author{
DOI: $10.46932 / \mathrm{sfjdv2n5-068}$
}

Received in: Oct 1st, 2021

Accepted in: Dec 30th, 2021

\author{
Pedro Daniel Astudillo Castro \\ Magister en Psicodiagnóstico y Evaluación Psicológica \\ Universidad de Azuay. Cuenca/Ecuador \\ E-mail: pedroastudillo1987@gmail.com \\ Christian Geovanny Quezada Ortega \\ Magister en Psicoterapia del Niño y la Familia \\ Instituto Superior Tecnológico del Azuay/ Ecuador \\ E-mail: christianquezadaortega@gmail.com
}

\section{RESUMEN}

El Inventario de Depresión de Beck (BDI-II) es uno de los instrumentos más empleados a nivel clínico e investigativo para evaluar la depresión. Objetivo. Adaptar y validar el BDI-II para su uso en la identificación de síntomas depresivos en población adolescente de Ecuador. Método. Estudio instrumental orientado a obtener la adaptación cultural, evidencias de validez y fiabilidad del (BDI-II). Participaron 871 adolescentes entre 13 y 18 años de edad seleccionados por muestreo no probabilístico. La validez del contenido del instrumento se evaluó mediante criterio de jueces y cálculo del Coeficiente de concordancia W de Kendall. Se realizó Análisis Factorial a través del Método de Componentes Principales. La asociación entre variables se analizó con el Coeficiente de correlación de Rho de Spearman. Para evaluar la validez convergente del (BDI-II) se empleó la Escala de Desesperanza de Beck (BHS) y la Escala de Valoración del Estado de Ánimo (EVEA). Resultados. El análisis factorial evidenció la existencia de una matriz de dos factores que explicó el $43.12 \%$ de la varianza total observada. Se obtuvieron evidencias favorables sobre validez y consistencia interna del instrumento $(a=.86)$. Conclusiones. La versión ecuatoriana del BDI-II posee estructura bifactorial consistente con la versión original y adaptaciones precedentes realizadas al instrumento en otros países de habla hispana. Es apto para ser utilizado en el diagnóstico de síntomas depresivos en población adolescente en el contexto ecuatoriano.

Palabras clave. Depresión, (BDI-II), adolescencia, adaptación, propiedades psicométricas.

\begin{abstract}
The Beck Depression Inventory (BDI-II) is one of the most widely used clinical and research instruments to assess depression. Objective. To adapt and validate the BDI-II for its use in the identification of depressive symptoms in the adolescent population of Ecuador. Method. Instrumental study oriented to obtain the cultural adaptation, validity and reliability evidences of the BDI-II. A total of 871 adolescents between 13 and 18 years of age selected by non-probabilistic sampling participated. The content validity of the instrument was evaluated by means of judges' criteria and calculation of Kendall's W concordance coefficient. Factorial analysis was performed using the Principal Component Method. The association
\end{abstract}


between variables was analyzed with Spearman's Rho correlation coefficient. The Beck Hopelessness Scale (BHS) and the Mood Rating Scale (EVEA) were used to evaluate the convergent validity of the BDI-II. Results. Factor analysis showed the existence of a two-factor matrix that explained $43.12 \%$ of the total variance observed. Favorable evidence was obtained on the validity and internal consistency of the instrument $(\mathrm{a}=.86)$. Conclusions. The Ecuadorian version of the BDI-II has a bifactorial structure consistent with the original version and previous adaptations made to the instrument in other Spanishspeaking countries. It is suitable for use in the diagnosis of depressive symptoms in the Ecuadorian adolescent population.

Keywords. Depression, (BDI-II), adolescence, adaptation, psychometric properties.

\section{INTRODUCCIÓN}

La adolescencia es una etapa del ciclo de vida comprendida entre los 10 y 19 años; es reconocida como un periodo de transición, de crecimiento y desarrollo humano, que deviene en la preparación del individuo para la adultez (Lozano, 2014; Barrionuevo, 2016). Más allá de la maduración física y psicológica que la caracteriza, destaca por ser una experiencia de cambios conducentes a la independencia económica y social, la definición de la identidad personal y la incorporación de aptitudes que posteriormente serán puestos a prueba en el desarrollo de las funciones del individuo adulto. Además, la adolescencia es también una época de potenciales riesgos, donde el contexto social puede tener una influencia determinante (OMS, 2015).

A lo largo de los años, la adolescencia se ha definido desde puntos de vista, psicológicos, antropológicos, históricos, sociales, sociológicos, donde no ha existido un punto de encuentro o de convergencia teórica al respecto. Según señalan García \& Parada (2018) y Jewell et al., (2019), las diferencias en los enfoques para concebir la adolescencia representan una invitación a hacer comparaciones, a contrastar unas posturas con otras, y a arribar a conclusiones que permitan alcanzar un posicionamiento individual que se ajuste y tenga utilidad práctica, teórica y metodológica en correspondencia al campo de conocimiento desde donde se aborde la adolescencia.

Tomando en cuenta lo anterior, se advierte la dificultad de enmarcar la adolescencia solamente considerando los cambios físicos y psicológicos evidentes, la misma debe ser considerada también una edad social y una construcción histórico-cultural que responde a las características de la época, el entorno y la sociedad. En el contexto de la presente investigación, se asume el criterio de que la adolescencia es un proceso de desarrollo, donde confluyen una multiplicidad de factores generadores de vulnerabilidad en los individuos, los cuales pueden experimentar diversas alteraciones, entre ellas, depresión (Contreras et al., 2015). 
Según los datos que versan en el informe de la Organización Mundial de la Salud (OMS), titulado "Salud para los adolescentes del mundo. Una segunda oportunidad en la segunda década"; la depresión constituye la principal causa de enfermedad y discapacidad entre los adolescentes de ambos géneros. Dicha organización hace un llamado de atención para que se proteja esta fase de la vida y señala la necesidad de garantizar un desarrollo estable y una buena situación de salud durante la adolescencia, ya que esto va a repercutir en la etapa adulta e incluso en la salud y el desarrollo de posteriores generaciones (OMS, 2014).

El primer episodio de depresión o el incremento exacerbado de síntomas depresivos, ocurre generalmente cuando el individuo es adolescente (Cairns et al., 2015). Datos actuales pronostican un incremento en la prevalencia de depresión en adolescentes a nivel mundial (Lu, 2019). En el Ecuador existe un aproximado de 1.419.537 adolescentes que representan el 9,8\% de la población y cinco de cada cien padecen de trastorno depresivo (INEC; 2016; 2021).

Se señala que la depresión en adolescentes es un fenómeno multifactorial (Bernaras, 2017; Zhao, 2021); un punto álgido dentro del abordaje del tema lo constituye su medición. En el Ecuador, específicamente, los instrumentos que se emplean en investigación, para la evaluación y diagnóstico de depresión en adolescentes no han sido sometidos a un proceso riguroso de validación que garantice la fiabilidad de los resultados que se obtienen (Astudillo, 2016; Abambari, 2013; Segovia et al., 2017; Mantilla, 2017). Por otro lado, los estudios de depresión realizados en adolescentes supuestamente sanos, presentan como limitaciones el uso de tamaños de muestra pequeños y el empleo de clasificaciones dicotómicas de la depresión (presencia-ausencia).

Es por el ello que el presente estudio se propone como objetivo general adaptar y validar el Inventario de Depresión de Beck-II para su uso en la identificación de la intensidad de síntomas depresivos en adolescentes del Ecuador. Para ello como objetivos específicos se plantean, obtener evidencias de validez de contenido, de constructo y convergente de la versión adaptada al Ecuador del Inventario de Depresión de Beck BDI-II y evaluar la consistencia interna del Inventario de Depresión de Beck BDI-II (adaptado).

\section{MÉTODO}

Se desarrolló un estudio instrumental para realizar la adaptación cultural y obtener evidencias de validez y fiabilidad del Inventario de Depresión de Beck (BDI-II). Se trabajó con una muestra no probabilística de 871 adolescentes entre 13 y 18 años de edad procedentes de Colegios seleccionados para 
el estudio, que se distribuyeron en correspondencia al cumplimiento de los objetivos propuestos ${ }^{1}$. Se contó con el consentimiento informado de los padres o representantes y el asentimiento de los adolescentes, autorizando la participación voluntariamente en la investigación. Se garantiza la confidencialidad y anonimato de los datos ofrecidos.

\subsection{PROCEDIMIENTOS}

La investigación se desarrolló en el contexto de instituciones educativas. Se explicó a los participantes el propósito de la investigación; los instrumentos fueron administrados a los adolescentes de los niveles educativos de Educación General Básica y Bachillerato, los cuales fueron presentados en forma de cuadernillo. La primera página contenía una ficha de datos generales elaborada ad hoc, seguido del Inventario de Depresión de Beck BDI-II, la Escala de Valoración del Estado de Ánimo (EVEA) y Escala de Desesperanza de Beck (BHS).

- Inventario de Depresión de Beck BDI-II (Beck, 1996; Adaptación argentina: Brenlla \& Rodríguez, 2006). Este instrumento permite la identificación y descripción de la gravedad de la sintomatología depresiva presente en el individuo. Para su uso se contó con el permiso de la Editorial Paidós de Buenos Aires y de los autores de dicha adaptación. Este instrumento consta de 21 ítems que a su vez se corresponden con síntomas depresivos: tristeza, pesimismo, fracaso, pérdida de placer, sentimientos de culpa, sentimientos de castigo, disconformidad con uno mismo, autocrítica, pensamientos o deseos suicidas, llanto, agitación, pérdida del interés, indecisión, desvalorización, pérdida de energía, cambios en los hábitos de sueño, irritabilidad, cambios de apetito, dificultad de concentración, cansancio o fatiga, pérdida de interés por el sexo.

- Escala de Valoración del Estado de Ánimo EVEA (Sanz, 2001; validada en Ecuador Aguirre, 2016). Es un instrumento que permite medir el estado de ánimo transitorio de un individuo en un momento dado. La EVEA es una técnica que consta de 16 ítems, cada uno compuesto por una escala gráfica de tipo Likert de 11 puntos (de 0 a 10), flanqueadas por las palabras "nada" (0) y "mucho" (10). Las 16 frases tienen la misma construcción; todas empiezan con las palabras "me siento" y continúan con un adjetivo que representa un estado de ánimo (p. ej., "me siento triste", "me siento alegre"). La EVEA evalúa cuatro estados de ánimo en particular: ansiedad, irahostilidad, tristeza-depresión y alegría. Es un instrumento que tiene una consistencia de $\alpha=.75$.

- Escala de Desesperanza-BHS (Beck, 1974; Adaptación argentina, Mikulic et al., 2009). Dicho instrumento evalúa pesimismo en las personas, considerando actitudes negativas que se refieren a

\footnotetext{
${ }^{1} 105$ adolescentes para prueba piloto de validez de contenido, 616 para el estudio de validez de constructo del instrumento y 150 para estudio de validez convergente.
} 
sí mismo y al futuro. La misma está compuesta por 20 proposiciones definibles por verdadero o falso que evalúan el alcance de las expectativas negativas referidas al futuro inmediato y a largo plazo. La confiabilidad del instrumento es de $\alpha=.73$.

Para el proceso de adaptación cultural y validez del contenido del instrumento se empleó la mencionada versión del BDI-II adaptado a la población argentina; a pesar de que el mismo se encuentra en castellano, existen peculiaridades lingüísticas y socioculturales en el territorio ecuatoriano, susceptibles de provocar dificultades en la comprensión del instrumento.

El análisis de validez de contenido del instrumento se desarrolló tanto de forma cualitativa mediante el criterio de jueces, como cuantitativa. Fueron seleccionados cinco jueces considerados expertos, tomando como criterios que fueran profesionales con experiencia mínima de cinco años en su área de conocimiento: 2 en Psicología, 1 en Psicometría, 1 en Adolescencia y 1 en Lingüística. Se aplicó el Método Delphi, con la finalidad de procurar obtener consenso en la opinión de los expertos sobre el instrumento (Urrutia et al., 2015). Además, se realizó una prueba piloto del instrumento.

El contacto con los jueces para orientar el proceso a desarrollar fue personal y a través de correo electrónico; no hubo vínculo entre ellos, lo cual constituye una de las bondades de este método, ya que se minimiza el impacto que pueden tener en un trabajo grupal los líderes de opinión. De esta forma cada experto tuvo la posibilidad de expresarse libremente. La concordancia entre el criterio de los jueces con respecto a la pertinencia, claridad de redacción y calidad conceptual de los ítems del Inventario, se calculó utilizando el Coeficiente de concordancia W de Kendall.

Para la validez del constructo se realizó un análisis factorial exploratorio (AFE), se empleó el método de extracción de Componentes Principales y la rotación Oblicua (Oblimin). Se calculó además el índice de adecuación maestral de Káiser-Mayer-Olkin (KMO) y se realizó la prueba de esfericidad de Barttlet. Se empleó el cálculo de coeficiente de correlación de Rho de Spearman, $\rho$ (rho) para el análisis de la validez convergente del Inventario BDI-II con escalas de medidas similares: Escala de Desesperanza de Beck (BHS) y las subescalas Tristeza-depresión y Ansiedad-alegría de la Escala de Valoración del Estado de Ánimo (EVEA). Adicionalmente, se utilizó el Coeficiente alfa de Cronbach para el análisis de consistencia interna de los instrumentos.

\section{RESULTADOS}

\subsection{VALIDEZ DE CONTENIDO DEL INSTRUMENTO}

Para obtener el criterio de jueces, primeramente, fueron explicadas las dimensiones del BDI-II y los indicadores para su evaluación. Se consideró para cada ítem, una valoración respecto a los indicadores: 
pertinencia, calidad de redacción y claridad conceptual. Se estableció el peso diferencial de los criterios de evaluación de cada ítem del instrumento mediante una escala Likert de 1 a 4 puntos, ascendente: (1) mala, (2) regular, (3) buena y (4) excelente.

Los expertos señalaron que la totalidad de los ítems del Inventario de Depresión de Beck BDI-II eran pertinentes y presentaban claridad conceptual; sin embargo, coincidieron en que la calidad de la redacción debía ser revisada para adecuarla al contexto sociocultural de la población en la que iba a ser empleado el instrumento. A partir del criterio de los jueces surge la necesidad de realizar algunos cambios en varios de los ítems del instrumento, conservándose el número original de estos. Fueron sugeridas transformaciones en 8 ítems que los expertos consideraron imprecisos y en otros que, según su criterio, contenían expresiones o términos ante los cuales los adolescentes ecuatorianos presentarían dificultades para comprender.

En el ítem 2, relacionado con el síntoma "Pesimismo" en el primer y segundo literales se realizaron los siguientes cambios de la afirmación original "No estoy desalentado respecto de mi futuro" fue cambiado a "No estoy desanimado respecto a mi futuro"; y de "Me siento más desalentado respecto de mí futuro que lo que solía estarlo" por "Me siento más desanimado respecto a mi futuro de lo que solía estar”. El ítem 3 "Fracaso" de "He fracasado más de lo que habría debido" cambió a "He fracasado más de lo que hubiera querido”. El Ítem 11. “Agitación” del original "No estoy más inquieto o tenso que lo habitual" cambió a "No estoy más inquieto o tenso de lo habitual"; de "Me siento más inquieto o tenso que lo habitual" cambió a "Me siento más inquieto o tenso de lo habitual”. En el ítem 15 "Pérdida de energía" originalmente la segunda afirmación señala, "No tengo suficiente energía para hacer demasiado." Fue sustituida por "No tengo suficiente energía para hacer demasiadas actividades”. Ítems 16, 17, 18 y 20 “Cambio en hábitos de sueños, Irritabilidad, Cambio de apetito y Cansancio o Fatiga” en varias afirmaciones que poseen la expresión “...más que lo habitual” y “... menos que lo habitual” se sugirió el cambio por “......más de lo habitual” y "menos de lo habitual”.

Adicionalmente, en la tabla 1 puede observarse que hubo cercanía en los rangos obtenidos en los tres indicadores empleados en las evaluaciones realizadas por los expertos; en el mencionado proceso de validación de contenido puede distinguirse que la $\mathrm{W}$ de Kendall ${ }^{\mathrm{a}}$ alcanzó un valor (.853). Los valores de W de Kendall ${ }^{\mathrm{a}}$ varían de ( 0 a 1$)$ donde el cero es señal de total desacuerdo entre los expertos y los valores más cercanos a uno hacen referencia a un total acuerdo entre los mismos (Escobar, 2008). Además, el nivel de significación de $(\mathrm{p}=.003<.05)$ señala que existe concordancia estadísticamente significativa entre los rangos asignados por los jueces expertos a cada indicador. 
Tabla 1 Indicadores y rangos del juicio de expertos en la evaluación de contenido del Inventario BDI-II y coeficiente de concordancia de Kendall

\begin{tabular}{lc}
\hline Indicadores & Rangos \\
\hline Pertinencia & 3.17 \\
Calidad de la redacción & 3.64 \\
Claridad conceptual & 3.19 \\
\hline $\mathrm{N}$ & 21 \\
\hline $\mathrm{W}$ de Kendall & $\mathrm{a}$ \\
$\mathrm{X}^{2}$ & 853 \\
gl & 64.417 \\
Sig. asintótica & 4 \\
\hline
\end{tabular}

\subsection{RESULTADOS DE PRUEBA PILOTO}

Posteriormente, el BDI-II fue sometido a una prueba piloto con 105 adolescentes de 13 a 18 años. El número de sujetos previsto para la prueba piloto obedece al criterio de autores que señalan que, para evaluar la consistencia de un instrumento, el número ideal para hacer prueba piloto es de 5 personas por ítem (Payares, 2016). Como resultado de este procedimiento fueron incorporados algunos nuevos cambios en el Inventario. Una de las dificultades que de manera reiterada fue presentada por los participantes, estuvo relacionada con la comprensión de la forma verbal "solía", que aparece en 12 oportunidades en el instrumento original. La misma fue sustituida en todos los ítems por el sinónimo "acostumbraba" que resultó ser más comprensible para los adolescentes; por ejemplo: "Tengo menos energía que la que solía tener" por "Tengo menos energía que la que acostumbraba tener." Sobre la base de los resultados de este proceso se confeccionó la versión final del Inventario de Depresión de Beck-II.

\subsection{VALIDEZ DE CONSTRUCTO DEL BDI-II ADAPTADO}

La versión final de instrumento se aplicó a 616 participantes. Con los datos recolectados en esta muestra de adolescentes se realizó primeramente un análisis factorial exploratorio sin tener definido a priori el número de factores con que se reduciría el conjunto de variables estudiadas; en este proceso se extrajeron cuatro factores. Posteriormente y tomando como referente los antecedentes teóricos y empíricos que respaldan la extracción de dos factores en el BDI-II (Osman et al., 2008; Wang et al.,2020), se realizó un segundo análisis exploratorio, en esta oportunidad, predeterminando la extracción de dos factores (Contreras, 2015). El fin de este procedimiento fue conocer los reactivos que se agruparían en uno u otro factor según la muestra estudiada.

Para desarrollar dicho procedimiento se calculó el índice de adecuación de la muestra KáiserMayer-Olkin, (KMO). También se realizó la prueba de esfericidad de Barttlet, cuyo valor fue $\mathrm{X}^{2}=$ $2825,680 / \mathrm{gl}=210$ y un valor de significancia $\mathrm{p}<.001$; dichos resultados indicaron la factibilidad de los datos para proceder con el análisis factorial del instrumento. La realización del AFE tuvo la intención de hacer una extracción del mínimo número de dimensiones o componentes que hicieran posible explicar la 
mayor cantidad de información que contienen los datos; es decir, que definieran mejor la estructura general de varianzas.

Al observarse la matriz de comunalidades que se muestra en la tabla 2, que ofrece datos sobre el promedio de correlación de cada variable que forma parte del instrumento, destaca que la mayoría de los valores de extracción son superiores a .39, lo cual es considerado de bueno a excelente, tomando como referente los criterios de Ebel y Frisbie (1986) que señalan que valores mayores a .30 indican un calidad buena y mayor a .40 excelente. Sin embargo, nótese que el ítem 18 “Cambios en apetito”, presenta carga factorial baja mostrando un limitado aporte al factor correspondiente, lo cual pone en cuestionamiento la utilidad de esta variable para el modelo.

Tabla 2 Matriz de comunalidades análisis factorial exploratorio del Inventario de Depresión de Beck BDI-II adaptado (Ecuador) $\mathrm{n}=616$

\begin{tabular}{|c|c|c|}
\hline \multicolumn{3}{|c|}{ Comunalidades } \\
\hline SÍNTOMAS & Inicial & Extracción \\
\hline 1 Tristeza & 1.000 & .411 \\
\hline 2 Pesimismo & 1.000 & .480 \\
\hline 3 Fracaso & 1.000 & .401 \\
\hline 4 Pérdida de placer & 1.000 & .486 \\
\hline 5 Sentimientos de culpa & 1.000 & .445 \\
\hline 6 Sentimientos de castigo & 1.000 & .610 \\
\hline 7 Disconformidad con uno mismo & 1.000 & .452 \\
\hline 8 Autocrítica & 1.000 & .346 \\
\hline 9 Pensamientos o deseos suicidas & 1.000 & .667 \\
\hline 10 Llanto & 1.000 & .557 \\
\hline 11 Agitación & 1.000 & .493 \\
\hline 12 Pérdida del interés & 1.000 & .425 \\
\hline 13 Indecisión & 1.000 & .451 \\
\hline 14 Desvalorización & 1.000 & .477 \\
\hline 15 Pérdida de energía & 1.000 & .458 \\
\hline 16 Cambios en los hábitos de sueño & 1.000 & .322 \\
\hline 17 Irritabilidad & 1.000 & .437 \\
\hline 18 Cambios de apetito & 1.000 & .224 \\
\hline 19 Dificultad de concentración & 1.000 & .489 \\
\hline 20 Cansancio o fatiga & 1.000 & .483 \\
\hline 21 Pérdida de interés por el sexo & 1.000 & .631 \\
\hline
\end{tabular}

En otro nivel de análisis de los resultados, está la información que brinda la varianza explicada que se muestra en la tabla 3 (se presenta incompleta ya que fue cortada para mostrar solo los datos que aportan información relevante para el presente análisis). Los componentes que genera el análisis factorial, explican una determinada cantidad de varianza de las respuestas observadas que definen la matriz de datos. En el caso de este análisis factorial exploratorio para el BDI-II adaptado, realizado sin haber definido a priori el número de factores, arrojó cuatro dimensiones con cargas de autovalores superiores a 
(1). Los primeros cuatro autovalores que se presentaron de forma consecutiva fueron $(5.9,1.2,1.1$ y 1.0$)$. Con estos cuatro componentes señalados se está explicando el $44.32 \%$ de la varianza total observada.

Tabla 3 Varianza total explicada en análisis factorial exploratorio del Inventario de Depresión de Beck BDI-II adaptado (Ecuador) $\mathrm{n}=616$

\begin{tabular}{cccccccccc} 
Componente & \multicolumn{3}{c}{ Autovalores iniciales } & \multicolumn{2}{c}{$\begin{array}{c}\text { Sumas de cargas al cuadrado de } \\
\text { la extracción }\end{array}$} & $\begin{array}{c}\text { Sumas de cargas al cuadrado de } \\
\text { la rotación }\end{array}$ \\
\cline { 2 - 11 } & Total & $\begin{array}{c}\% \text { de } \\
\text { varianza }\end{array}$ & $\begin{array}{c}\% \\
\text { Acumulado }\end{array}$ & Total & $\begin{array}{c}\% \text { de } \\
\text { varianza }\end{array}$ & $\begin{array}{c}\% \\
\text { Acumulado }\end{array}$ & $\begin{array}{c}\text { Total } \\
\% \text { de } \\
\text { varianza }\end{array}$ & $\begin{array}{c}\% \\
\text { Acumulado }\end{array}$ \\
\hline $\mathbf{1}$ & 5.945 & 28.311 & 28.311 & 5.945 & 28.311 & 28.311 & 2.814 & 13.402 & 13.402 \\
\hline $\mathbf{2}$ & 1.219 & 5.805 & 34.115 & 1.219 & 5.805 & 34.115 & 2.758 & 13.132 & 26.534 \\
\hline $\mathbf{3}$ & 1.139 & 5.424 & 39.539 & 1.139 & 5.424 & 39.539 & 2.228 & 10.609 & 37.143 \\
\hline $\mathbf{4}$ & 1.004 & 4.779 & 44.318 & 1.004 & 4.779 & 44.318 & 1.507 & 7.175 & 44.318 \\
\hline $\mathbf{5}$ & .952 & 4.532 & 48.850 & & & & & & \\
\hline
\end{tabular}

A continuación, en la tabla 4 se presenta la matriz de componente rotado con el método de extracción de análisis de componentes principales. En ella se muestra la distribución de la varianza total observada entre los cuatro componentes antes mencionados, permitiendo entonces, que cada uno de ellos logre agrupar aquellas variables que están altamente correlacionadas entre sí. Nótese como dato relevante que, a partir de las correlaciones entre las variables, se generaron grupos de ítems en los componentes 1, 2, 3 y 4. En el primero se agrupan los ítems (11-Agitación; 13-Indesición; 12- Pérdida de interés; 20 Cansancio o fatiga, 17-Iritabilidad, 15 Pérdida de energía y 19-Dificultad de concentración). Dichos ítems, en su mayoría no se asocian con ningún otro componente, exceptuando el ítem 19, que presenta complejidad factorial, es decir, el mismo está compartiendo la carga en dos factores y su ubicación se define a partir del marco referencial. Si se analiza teóricamente esta agrupación puede decirse que la misma no es coherente desde el punto de vista teórico; en ella se han mezclado indistintamente tanto síntomas de depresión como somáticos y afectivos.

El componente dos agrupa los ítems (9-Pensamientos o deseos suicidas; 14-Desvalorización; 2Pesimismo; 1-Tristeza; 3-Fracaso, 19-Dificultad de concentración y 10- Llanto) de los cuales, cinco están cargando solamente en una dimensión; sin embargo, destacan el ítem 9 y el ya mencionado ítem 19, que presentan complejidad factorial. Puede decirse que este componente es más coherente desde el punto de vista teórico. En el caso del tercer componente se incluyen los ítems (6-Sentimientos de castigo; 4-Pérdida de placer y 5-Sentimientos de culpa). En el cuarto y último factor se encuentran los ítems (9-Pensamientos o deseos suicidas; 21 - Pérdida de interés por el sexo y 16-Cambios en los hábitos de sueño). Obsérvese, además, que los ítems (7-Disconformidad con uno mismo y 18- Cambios de apetito) no correlacionaron con ningún componente. 
Tabla 4 Matriz de componente rotado en análisis factorial exploratorio del Inventario de Depresión de Beck BDI-II adaptado (Ecuador) $\mathrm{n}=616$

\begin{tabular}{|c|c|c|c|c|}
\hline \multicolumn{5}{|c|}{ Matriz de componente rotado ${ }^{a}$} \\
\hline & \multicolumn{4}{|c|}{ Componente } \\
\hline & 1 & 2 & 3 & 4 \\
\hline 11 Agitación & .678 & & & \\
\hline 13 Indecisión & 649 & & & \\
\hline 12 Pérdida del interés & .577 & & & \\
\hline 20 Cansancio o fatiga & .506 & & & \\
\hline 17 Irritabilidad & .475 & & & \\
\hline 15 Pérdida de energía & .459 & & & \\
\hline 9 Pensamientos o deseos suicidas & .640 & & & .479 \\
\hline 14 Desvalorización & & .588 & & \\
\hline 2 Pesimismo & & .577 & & \\
\hline 1 Tristeza & & .572 & & \\
\hline 3 Fracaso & & .514 & & \\
\hline 19 Dificultad de concentración & .479 & .490 & & \\
\hline 8 Autocrítica & & .404 & & \\
\hline \multicolumn{5}{|l|}{7 Disconformidad con uno mismo } \\
\hline 6 Sentimientos de castigo & & & .766 & \\
\hline 4 Pérdida de placer & & & .616 & \\
\hline 5 Sentimientos de culpa & & & .579 & \\
\hline 10 Llanto & & .401 & & \\
\hline 21 Pérdida de interés por el sexo & & & & .775 \\
\hline 16 Cambios en los hábitos de sueño & & & & .447 \\
\hline \multicolumn{5}{|l|}{18 Cambios de apetito } \\
\hline $\begin{array}{l}\text { Método de extracción: análisis de co } \\
\text { Método de rotación: Oblimin con no }\end{array}$ & $\begin{array}{l}\text { rincipa } \\
\text { Kaiser }\end{array}$ & & & \\
\hline
\end{tabular}

Una vez realizado el anterior análisis el instrumento fue sometido a un segundo AFE, en esta ocasión a partir del modelo de dos factores que ha sido definido en estudios precedentes por Beck et al., (1996), como "Somático-afectivo y Cognitivo (Sa-C)"; Strunk \& Lane (2017), los denominaron (Somático y Cognitivo-afectivo (S-Ca) y de Sanz et al. (2003, 2009) los clasificaron como (Cognitivoafectivo y Somático-motivacional (Ca-Sm). En dicho análisis no puede perderse de vista el hecho de que existen ligeras diferencias en las estructuras que muestran los mencionados modelos. Este segundo procedimiento se realizó a partir de los datos que ofrece la matriz de componentes rotados del instrumento explicando el $43.12 \%$ de la varianza total observada, lo cual es adecuado según plantean Ferrando \& Carrasco (2010); estos autores hacen alusión a que se requieren soluciones factoriales que logren explicar por lo menos un $40 \%$ de la varianza total de las respuestas estudiadas en el número de componente que sean pertinentes y susceptibles de ser explicados teóricamente. Como puede observarse en el análisis realizado se superó el porcentaje mencionado.

El primer resultado que se observa en la tabla 5 es que en ambos factores las variables agrupadas están correlacionadas con valores superiores a (.40). La tabla muestra que en el primer factor se agruparon trece de los 21 síntomas que contiene el instrumento: pesimismo, fracaso, tristeza, desvalorización, pérdida de placer, sentimientos de castigo, disconformidad con uno mismo, sentimientos de culpa, irritabilidad, autocrítica, pensamientos o deseos suicidas, dificultad de concentración y llanto. De ellos 
hay cuatro que presentan las cargas más altas a este factor, con valores de (.60 a .70). La mayoría de los ítems agrupados en este primer factor hacen alusión a síntomas de depresión de tipo afectivos y motivacionales. Nótese además que la irritabilidad y la dificultad de concentración, comparten cargas en el factor 1 y 2. En el caso del segundo factor el síntoma "cansancio o fatiga" es el que presenta la mayor carga, siendo de (.64). En el mismo se agruparon 7 ítems: agitación, pérdida del interés, cambios en los hábitos de sueño, pérdida de interés por el sexo, indecisión, dificultad de concentración y pérdida de energía. En dicha agrupación pueden observarse síntomas de tipo somáticos y cognitivos. El ítem 18, correspondiente al síntoma "cambios en el apetito", no presentó carga en ninguno de los dos factores.

Tabla 5 Matriz de componente rotado en análisis factorial exploratorio de dos factores del Inventario de Depresión de Beck BDI-II adaptado (Ecuador) $\mathrm{n}=616$

\begin{tabular}{|c|c|c|}
\hline & 1 & 2 \\
\hline 2 Pesimismo & .679 & \\
\hline 3 Fracaso & .609 & \\
\hline 1 Tristeza & .604 & \\
\hline 14 Desvalorización & .601 & \\
\hline 4 Pérdida de placer & .581 & \\
\hline 6 Sentimientos de castigo & .571 & \\
\hline 7 Disconformidad con uno mismo & .570 & \\
\hline 5 Sentimientos de culpa & .496 & \\
\hline 17 Irritabilidad & .483 & .433 \\
\hline 8 Autocrítica & .445 & \\
\hline 9 Pensamientos o deseos suicidas & .428 & \\
\hline 20 Cansancio o fatiga & & .643 \\
\hline 11 Agitación & & .555 \\
\hline 12 Pérdida del interés & & .519 \\
\hline 16 Cambios en los hábitos de sueño & & .518 \\
\hline 21 Pérdida de interés por el sexo & & .502 \\
\hline 13 Indecisión & & .500 \\
\hline 19 Dificultad de concentración & .405 & .458 \\
\hline 15 Pérdida de energía & & .443 \\
\hline 10 Llanto & .401 & \\
\hline 18 Cambios de apetito & & \\
\hline
\end{tabular}

\subsection{VALIDEZ CONVERGENTE DEL BDI-II ADAPTADO}

Con el fin de estudiar la validez convergente del instrumento adaptado se realizaron correlaciones de éste con escalas de medidas con los que la depresión debería correlacionar: Escala de Desesperanza de Beck (BHS) y las subescalas Tristeza-depresión y Ansiedad-alegría de la Escala de Valoración del Estado de Ánimo (EVEA), las cuales fueron administradas a una muestra de 150 adolescentes. La tabla 6 ofrece los datos de las correlaciones entre la puntuación total del Inventario de Depresión de Beck BDI-II adaptado para Ecuador y las de la Escala de Valoración del Estado de Ánimo (EVEA) (Sanz, 2001) y la Escala de Desesperanza de Beck (BHS) Adaptación: (Mikulic et al., 2009). 
Dicho procedimiento se llevó a cabo para observar la existencia de correlaciones entre los instrumentos utilizados, para demostrar que el BDI-II adaptado en el presente estudio, constituye una buena medida del constructo depresión. Se realizó la correlación a partir del método de correlación de Spearman, $\rho$ (rho) debido a la distribución no normal de los datos. La tabla 6 ofrece los resultados obtenidos que muestran la existencia de una correlación estadísticamente significativa (rho $=.627^{*} *$, p=.001<.01) entre el Inventario de Depresión de Beck BDI-II adaptado para su uso en Ecuador y la Escala de Desesperanza de Beck (BHS). La correlación con la sub-escala de tristeza-depresión del EVEA, presentó resultados similares con $\left(\right.$ rho $\left.=564^{* *}, p=.002<.01\right)$. En cambio, obsérvese la asociación obtenida entre el BDI-II adaptado con la sub-escala de ansiedad-alegría del EVEA, la cual es baja y además no es estadísticamente significativa ( $\mathrm{rho}=.052, \mathrm{p}=.196>.01$ ). Dichos resultados confirman la validez convergente del Inventario de Depresión de Beck BDI-II adaptado en población adolescente ecuatoriana.

Tabla 6 Correlaciones entre el Inventario de Depresión de Beck BDI-II adaptado (Ecuador) y las escalas seleccionadas n=616 Escalas Rho de Spearman

\begin{tabular}{lcc}
\hline $\begin{array}{l}\text { Escala de Desesperanza de Beck } \\
\text { (BHS) }\end{array}$ & $.627^{* *}$ & .001 \\
\hline $\begin{array}{l}\text { Escala de Valoración del Estado de } \\
\text { Ánimo (EVEA) Tristeza-depresión }\end{array}$ & $.564^{* *}$ & .002 \\
\hline $\begin{array}{l}\text { Escala de Valoración del Estado de } \\
\text { Ánimo (EVEA) Ansiedad-alegría }\end{array}$ & .052 & .196 \\
\hline
\end{tabular}

\subsection{CONSISTENCIA INTERNA DEL BDI-II ADAPTADO}

El estudio de la consistencia interna del instrumento se realizó mediante el cálculo del Coeficiente Alfa de Cronbach, el valor obtenido fue $\alpha=.86$.

\section{DISCUSIÓN}

El presente estudio se propuso adaptar y validar el Inventario de Depresión de Beck- BDI-II para su uso en la identificación de síntomas depresivos en adolescentes de Ecuador. El primer objetivo específico ha sido estudiar la validez de contenido, lo cual se realizó de manera cualitativa mediante el criterio de jueces expertos y cuantitativa a través del cálculo del Coeficiente de concordancia W de Kendal. En relación a lo anterior puede señalarse que se alcanzó una concordancia en el criterio de jueces al evaluar la pertinencia, la calidad de redacción y la claridad conceptual de los ítems del Inventario, aspecto que se confirmó con el valor obtenido en el cálculo del coeficiente W de Kendalla ${ }^{\mathrm{a}}$, de (.853). Los jueces sugirieron adecuaciones al instrumento en correspondencia al contexto sociocultural de la población en la que va a ser empleado el instrumento.

También se realizó el análisis de la validez de constructo de la versión adaptada al Ecuador BDIII. A partir de un análisis factorial exploratorio inicial se extrajeron cuatro factores que explicaron el 
$44.32 \%$ de la varianza total observada. Respecto a este elemento, observa en los resultados que, en la distribución de la sintomatología depresiva, los síntomas, tanto somáticos como afectivos, se agruparon de una manera incoherente, lo cual dificulta la explicación de esas agrupaciones desde una postura teórica. Es por ello que se decide posteriormente, al realizarse un análisis factorial exploratorio tomando como referente el modelo de dos factores. Con dicho procedimiento se alcanzó un resultado similar al obtenido de manera sistemática en experiencias empíricas anteriores de estudios desarrollados por: Beck et al., (1996); Amaro (2015) en Perú; Contreras (2015) en México; Lipps, et al., (2077) en Jamaica; Sanz (2013) en España.

En la presente investigación se extrajeron de manera satisfactoria dos factores, los que fueron clasificados como "afectivo- motivacional" y "cognitivo-somático" que explicaron el 43.12\% de la varianza observada, lo cual resulta adecuado (Ferrando \& Carrasco, 2010); en cada factor las variables agrupadas están correlacionadas con valores superiores a (.40). Si bien existe similitud en cuanto a la estructura bifactorial obtenida con lo reportado en estudios precedentes, cabe señalar que se encontraron diferencias en la composición factorial. Al compararse tales resultados con los obtenido por Brenlla \& Rodríguez (2006) en Argentina en la validación del BDI-II en pacientes y en población general, se observa que un primer factor configura una dimensión "Cognitivo-Afectiva" de la depresión y los síntomas agrupados en el segundo factor describen, en su mayoría, una dimensión "Somática".

Este hallazgo concuerda con lo planteado por Osman et al., (2008) quienes señalan que el modelo de dos factores de Beck et al., se ha replicado solamente de manera parcial en otras investigaciones. El motivo fundamental atribuido a las diferencias en la composición factorial, es la diversidad de las características de las poblaciones con las que se ha estudiado la validez factorial del instrumento. Beck et al. (1996) señalaban que existían discrepancias en los resultados de los análisis del inventario, cuando se realizaban estudios con población general y cuando se hacían con población clínica.

Según este criterio documentado por Beck, para la discusión de este resultado, se decidió seleccionar entre los estudios precedentes el realizado por Contreras (2015) en población adolescente, el cual posee una mayor similitud con las características de la población con la que se trabajó en la presente investigación. Contreras (2015) obtuvo un modelo de dos factores en una población de adolescentes mexicanos. El análisis comparativo entre el mencionado estudio y esta investigación marcó diferencias en cuanto a la cantidad de ítems agrupados por factores y los síntomas depresivos en cada uno de ellos. En el estudio de Contreras (2015), el factor 1 cuenta con 16 ítems de naturaleza "afectivo-cognitiva" y el segundo factor tiene 5 ítems correspondientes a la sintomatología "somático-vegetativa" de la depresión.

En el presente trabajo investigativo, el primer factor agrupa 13 síntomas y el factor 2, un total de seis; además, hubo un ítem que no presentó carga en ninguno de los dos factores. En cuanto al tipo de 
síntomas, puede decirse que en factor 1 se agruparon mayoritariamente de tipo afectivo-motivacional en el factor cognitivo-somático. Se comprobó que, a pesar de haberse empleado el mismo instrumento en poblaciones con características demográficas similares, las estructuras factoriales encontradas fueron diferentes. Al contrastarse los resultados de estos estudios, donde es evidente una marcada diferencia en la distribución de ítems por factor en cada análisis realizado, una de las conclusiones a las que puede arribarse es que resulta muy difícil replicar una estructura factorial única. En términos tanto investigativos como de la práctica clínica, esto sería un fin deseable, pero desde la evidencia empírica recolectada hasta el momento, no ha sido posible.

Del Pino et al., (2012) al referirse a este aspecto, hace alusión a dos posiciones contrapuestas; por un lado plantea la necesidad de aceptar la variabilidad relativa de la estructura del BDI-II, atribuyéndole esta característica al instrumento como algo propio, y por otro, se insta a los investigadores a realizar estudios psicométricos a partir del planteamiento de modelos con un nivel de complejidad más elevado, con análisis factoriales confirmatorios que sean capaces de ajustarse a su vez a la propia complejidad que también posee el constructo depresión. Relacionado a lo anterior, resulta necesario que también se tome en cuenta la importancia de adaptar el instrumento a cada contexto cultural, optimizando la validez ecológica del mismo y su posibilidad de ser aplicado en poblaciones con características similares a la de la adaptación (Ram et al., 2017).

También se obtuvieron evidencias favorables sobre la validez convergente del instrumento, dado que se han registrado correlaciones entre las medidas del BDI-II adaptado, la Escala de Desesperanza de Beck (BHS) y las subescalas tristeza-depresión y ansiedad-alegría de la Escala de Valoración del Estado de Ánimo (EVEA). Un segundo objetivo de este estudio estuvo orientado a evaluar la consistencia interna de la versión adaptada al Ecuador del Inventario. En este nivel de análisis puede mencionarse que todas las versiones del BDI-II han demostrado tener una buena consistencia interna. En la presente investigación también se constató lo anterior, el alfa de Cronbach alcanzado por el instrumento es de $(a=.86)$. $\mathrm{Al}$ comparase con otros estudios se advierte similitud en los resultados obtenidos lo que ha demostrado sistemáticamente que el BDI-II es un instrumento confiable. (Brenlla \& Rodríguez, 2006; Sanz, 2013; Amaro, 2015; Strunk, 2017; Wang, 2020).

\section{CONCLUSIONES}

Se obtuvo una versión adaptada del Inventario de Depresión de Beck BDI-II para ser utilizado en el diagnóstico de síntomas depresivos en población adolescente de Ecuador. Desde el punto de vista psicométrico puede decirse que la versión adaptada del instrumento reprodujo la estructura bifactorial 
original, compuesta por un factor afectivo-motivacional y otro cognitivo-somático. Además, mostró evidencias favorables de validez de contenido, de constructo, convergente y consistencia interna. 


\section{REFERENCIAS}

Abambari, C. Z. (2013). La disfuncionalidad familiar como factor asociado a Depresión y Ansiedad en la población adolescente de Santa Isabel. Revista Médica HJCA, 5(1), 69-73. Obtenido de https://revistamedicahjca.iess.gob.ec/ojs/index.php/HJCA/article/view/475

Aguirre, G. A. (2916). Violencia intrafamiliar y su influencia en el estado de ánimo de los estudiantes del Primero de Bachillerato Ciencias Básicas sección vespertina del Colegio Técnico Fiscal mixto 27 de Febrero de la ciudad de Loja. Universidad Nacional de Loja. Obtenido de https://dspace.unl.edu.ec/jspui/handle/123456789/11302?mode=full

Amaro, E. A. (2015). Propiedades Psicométricas del Inventario de Depresión de Beck en estudiantes de 5to año de secundaria de Huancayo, 2013. . Revista de Investigación Universitaria, 4(2).

Astudillo, S. M. (2016). Frecuencia de ansiedad y depresión en niñas y adolescentes de la casa de acogida" Miguel León" del Cantón Cuenca-Ecuador, 2016. Bachelor's thesis. Obtenido de http://dspace.ucuenca.edu.ec/handle/123456789/25503

Barrionuevo, J. (2016). Adolescencia y juventud. Buenos Aires: EUDEBA. Obtenido de https://books.google.es/books?hl=es\&lr=\&id=QChrDQAAQBAJ\&oi=fnd\&pg=PT7\&dq=definici\%C3\% $\mathrm{B} 3 \mathrm{n}+\mathrm{de}+$ adolescencia\&ots $=\mathrm{wCzJhYiaPM} \& \operatorname{sig}=7 \mathrm{ttf92}-$

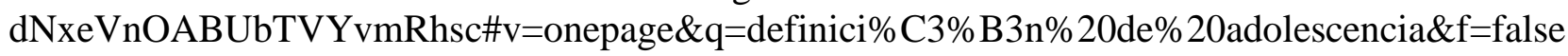

Bernaras, E. J. (2017). Desajustes escolar y clínico, y ajuste personal de adolescentes de 12 a 18 años. Revista de Psicodidáctica, 22(2), 118-127. Obtenido de https://www.sciencedirect.com/science/article/abs/pii/S1136103417300977

Bernaras, E. J. (2019). Child and adolescent depression: a review of theories, evaluation instruments, prevention programs, and treatments. Frontiers in psychology, 10, 543. Obtenido de https://internaljournal.frontiersin.org/articles/10.3389/fpsyg.2019.00543/full

Brenlla, M. E. (2006). Adaptación argentina del Inventario de Depresión de Beck (BDI-II). BDI-II. Inventario de depresión de Beck, . Buenos Aires, Argentina: Paidós.

Cairns, K. E. (2015). Identifying prevention strategies for adolescents to reduce their risk of depression: A Delphi consensus study. . Journal of Affective Disorders, 183,, 229-238. Obtenido de https://pubmed.ncbi.nlm.nih.gov/26025369/

Contreras, V. J.-G. (2015). Validez de constructo del Inventario de Depresión de Beck II para adolescentes. . Terapia Psicologica, 33(3), , 195-203. Obtenido de https://doi.org/10.4067/S071848082015000300004

Contreras-Valdez, J. A.-G. (2015). Validez de constructo del Inventario de Depresión de Beck II para adolescentes. Terapia Psicologica, 33(3), 195-203. Obtenido de https://doi.org/10.4067/S071848082015000300004

Del Pino, A. D. (2012). Modelos factoriales del Inventario de Depresión de Beck-II. Validación con pacientes coronarios y una crítica al modelo de Ward. Psicothema, 24(1), 127-132. Obtenido de http://www.psicothema.com/psicothema.asp?id=3989 
Escobar-Pérez, J. \&.-M. (2008). Validez de contenido y juicio de expertos: una aproximación a su utilización. Avances en medición, 6(1), 27-36. Obtenido de https://dialnet.unirioja.es/servlet/articulo?codigo=2981181

Ferrando, P. J.-C. (2010). El análisis factorial como técnica de investigación en psicología. Papeles del psicólogo, 31(1). Obtenido de https://www.redalyc.org/pdf/778/77812441003.pdf

García, C. I. (2018). Construcción de adolescencia: una concepción histórica y social inserta en las políticas públicas. Universitas humanística, (85). Obtenido de https://revistas.javeriana.edu.co/index.php/univhumanistica/article/view/22490/

Instituto Nacional de Estadísticas y Censos (INEC). . (2016). Principales causas de morbilidad. Compendio estadístico. Compendio estadístico digital. Quito, Ecuador. . Obtenido de http://www.ecuadorencifras.gob.ec/documentos/web

Instituto Nacional de Estadísticas y Censos (INEC). (2021). Cuentas satélites de salud. Notas técnicas de mejoras matodológicas. Gestion de análisis de síntesis. Obtenido de https://www.ecuadorencifras.gob.ec/documentos/web-

inec/Estadisticas_Economicas/Cuentas_Satelite/Cuentas_Satelite_2007-

2019/7.1_Nota_tecnica_CSS.pdf

Jewell, T. G. (2019). Attachment measures in middle childhood and adolescence: A systematic review of measurement properties. Clinical psychology review, 68, 71-82. Obtenido de https://www.sciencedirect.com/science/article/pii/S027273581830326X

Lipps, G. E. (2017). Validation of the beck depression inventory-II in a Jamaican university student cohort., 404-408. West Indian Medical Journal, 56(5). Obtenido de https://www.researchgate.net/publication/5548249_Validation_of_the_Beck_Depression_InventoryII_in_a_Jamaican_University_student_cohort

Lozano, V. A. (2014). Teoría de teorías sobre la adolescencia. Última década, 22(40), 11-36. Obtenido de https://scielo.conicyt.cl/scielo.php?pid=S0718-22362014000100002\&script=sci_arttext

Lu, W. (2019). “Adolescent Depression: National Trends, Risk Factorsy Healthcare Disparities". American journal of health behavior, 43(1), 181-194. Obtenido de https://www.ingentaconnect.com/content/png/ajhb/2019/00000043/00000001/art00015

Mantilla, F. L. (2017). La disfuncionalidad familiar y los niveles de depresión en adolescentes de una unidad educativa rural en Ambato, Ecuador. Revista Eniac Pesquisa, 6(2), , 315-324.

Mikulic, I. M. (2009). Escala de Desesperanza BHS (A. Beck, 1974): estudio de las propiedades psicométricas y baremización de la Adaptación Argentina. . Anuario de investigaciones, 16,, 365-373.

Morla Boloña, A. \&. (2006). Depresión en adolescentes y desestructuración familiar en la ciudad de Guayaquil, Ecuador. Revista Colombiana de Psiquiatría, XXXV,. 149-166. Obtenido de http://www.redalyc.org/pdf/806/80635203.pdf

Organización MUndial de la Salud (OMS). (2014). Salud para los adolescentes del mundo. Una segunda oportunidad en la segunda década. Oms, 1, 20. Obtenido de www.who.int/adolescent/second-decade 
Osman, a. B. (2008). (Psychometric properties of the Beck Depression inventory-ii in nonclinical adolescent samples. Journal of Clinical Psychology, 64. Obtenido de https://pubmed.ncbi.nlm.nih.gov/18161034/

Ram, N. B. (2017). The questionable ecological validity of ecological momentary assessment: Considerations for design and analysis. Research in Human Development, 14(3), 253-270. Obtenido de https://www.tandfonline.com/doi/abs/10.1080/15427609.2017.1340052

Sanz, J. (2013). 50 Years of the Beck Depression Inventories: Recommendations for the Use of the Spanish Adaptation of the BDI-II in Clinical, 1-28. Papeles del Psicólogo 34(3), 161-168. Obtenido de https://scholar.google.com.ec/scholar?

Sanz, J. y.-V. (2009). The Beck Depression Inventory-second edition (BDI-II): factor congruence and generalizability of its indexes of internal consistency. . Řehulka .

Segovia, P. M. (2017). Depresión en adolescentes escolarizados de décimo año de la Unidad Educativa Municipal Sebastián de Benalcázar de la Ciudad de Quito de la Provincia de Pichincha. Universidad Central de Ecuador. Obtenido de http://www.dspace.uce.edu.ec/handle/25000/9854

Strunk, K. K. (2017). The Beck Depression Inventory, (BDI-II): A cross-sample structural analysis. . Measurement and Evaluation in Counseling and Development, 50(1-2), 3-17.

Urrutia, M. B. (2015). Métodos óptimos para determinar validez de contenido. Revista Cubana de Educacion Medica Superior, 28(3), $\quad$ 547-558. Obtenido de http://scielo.sld.cu/scielo.php?script=sci_arttext\&pid=S0864-21412014000300014

Wang, X. C. (2020). The Psychometric Properties of the Chinese Version of the Beck Depression Inventory-II With Middle School Teachers. Measurement and Evaluation in Counseling and Development, 41, 13-31. Obtenido de https://www.frontiersin.org/articles/10.3389/fpsyg.2020.548965/full

Zhao, M. (2021). The Role of Psychosocial Factors in Adolescent Depressive Symptoms. University of Exeter. Obtenido de https://ore.exeter.ac.uk/repository/handle/10871/126886 